\title{
Nocardiopsis mutabilis, a New Species of Nocardioform Bacteria Isolated from Soil
}

\author{
MARCIA C. SHEARER, ${ }^{1 *}$ PAULA M. COLMAN, ${ }^{1}$ AND CLAUDE H. NASH III ${ }^{2}$ \\ Department of Natural Products Pharmacology, Smith Kline \& French Laboratories, Philadelphia, \\ Pennsylvania $19101^{1}$ and Sterling-Winthrop Research Institute, Rensselaer, New York $12144^{2}$
}

\begin{abstract}
A nocardioform bacterium isolated from soil was studied. On the basis of cell wall composition and physiological characteristics, this organism was placed in the genus Nocardiopsis. This organism differed from the only previously described species of this genus by a number of morphological and biochemical characteristics, including inability to decompose adenine and xanthine, fragmentation in submerged cultures, growth in lysozyme broth, and possession of type PIV phospholipids. It also produced a novel antibiotic, polynitroxin. This organism is regarded as a new species, for which we propose the name Nocardiopsis mutabilis. The type strain of this species is SK\&F-AAA025 (= ATCC 31520).
\end{abstract}

Since instituting analysis of whole-cell hydrolysates as a routine part of our preliminary taxonomic studies, we have found a number of antibiotic-producing actinomycetes which contain meso-diaminopimelic acid and have a type C sugar pattern (13). Analyses of purified cell wall preparations from these organisms have revealed type III cell walls with no characteristic sugars (13). A few of these cultures have very distinct morphological characteristics which make them easy to assign to a genus. Others, however, have morphologies which fall somewhere in that continuous spectrum of morphological characteristics that links Streptomyces with Nocardia. At present, these actinomycetes are very difficult to classify because a very limited number have been isolated and adequately characterized. In this paper we describe the taxonomic characteristics of one of these actinomycetes, strain SK\&F-AAA025 (type strain), which produces a novel antibiotic (polynitroxin). The isolation and characterization of the antibiotic have been described elsewhere (T. C. Jain, D. J. Newman, and M. C. Shearer, U.S. patent 4,317,812, March 1982).

\section{MATERIALS AND METHODS}

Bacterial strain and culture methods. Strain SK\&FAAA $025^{\mathrm{T}}$ was isolated by standard soil dilution and plating techniques from a soil sample taken from a cultivated field in Gujarat, India.

Stock cultures were grown on medium 172 (M172) (1). Slants of this medium were also used for determining the growth temperature range. The media used for morphological observations were M172, thin potatocarrot agar (TPC) (6), and soil extract agar. The soil extract agar contained $1.0 \mathrm{~g}$ of dextrose, $0.2 \mathrm{~g}$ of $\mathrm{K}_{2} \mathrm{HPO}_{4}, 1,000 \mathrm{ml}$ of soil extract, and $20 \mathrm{~g}$ of agar $(\mathrm{pH}$ 7.0). The soil extract was prepared by adding $500 \mathrm{~g}$ of fertile field soil to $1,200 \mathrm{ml}$ of tap water, autoclaving this preparation for $30 \mathrm{~min}$, and filtering it through paper. Additional media used to characterize strain SK\&F-AAA025 ${ }^{\mathrm{T}}$ were yeast extract-malt extract agar (21), oatmeal agar (21), inorganic salts-starch agar (21), glycerol-asparagine agar (21), tyrosine agar (21), peptone-yeast extract-iron agar (21), tryptone-yeast extract broth (21), defined agar (9), glucose-yeast extract agar (24), gelatin medium 19 (24), Czapek-sucrose agar (Czapek-Dox broth [Difco Laboratories] solidified with agar), nutrient agar (Difco), litmus milk medium (Difco), and D Nase Test Agar (BBL Microbiology Systems).

All tests were performed at $28^{\circ} \mathrm{C}$. For growth tests under anaerobic conditions, the GasPak system (BBL) was used.

Microscopy. Whole mounts of sporophores were prepared by the method of Lechevalier and Lechevalier (12) and were viewed with a Siemens model $1 \mathrm{~A}$ transmission electron microscope.

Chemotaxonomy. Purified cell walls and whole-cell hydrolysates were analyzed by the methods of Becker et al. (2) and Lechevalier (10), respectively. Cell wall phospholipid and mycolic acid analyses were performed by the methods of Lechevalier et al. $(11,14)$.

Physiological tests. The physiological tests used to characterize strain SK\&F-AAA025 ${ }^{\mathrm{T}}$ were those of Gordon $(3,4)$ and Gordon and Mihm (5). In the tests for acid production from carbohydrates and utilization of organic acids, all results were confirmed by subculturing onto fresh medium. The tests used for melanoid pigments were those of Shirling and Gottlieb (21).

Susceptibility to penicillin and rifampin. The susceptibility of strain SK\&F-AAA025 $5^{\mathrm{T}}$ to pencillin and rifampin was examined by the following method: susceptibility disks (BBL) were placed on M172 plates seeded with a suspension of strain SK\&F-AAA025 
and after incubation for 1 week at $28^{\circ} \mathrm{C}$, the diameters of the zones of inhibition were measured.

\section{RESULTS}

General description. Strain SK\&F-AAA025 ${ }^{\mathrm{T}}$ was gram positive and not acid-fast. It was a filamentous organism that formed a mycelium differentiated into (i) a substrate mycelium that penetrated the agar and formed a compact layer on top of the agar and (ii) an aerial mycelium originating from the substrate mycelium. No motile elements were observed.

Colony morphology. A great variety of colony topography and texture was observed, particularly on rich organic media. There were two basic colony types, but a continuous spectrum of intermediate colony types was also usually present. One basic colony type was light yellow, butyrous, and convex with a smooth surface; this type of colony never produced any visible aerial mycelium. The second basic colony type was yellowish brown, leathery, and conical crateriform; this second colony type became completely covered with a white aerial mycelium. When well-isolated colonies of each type were

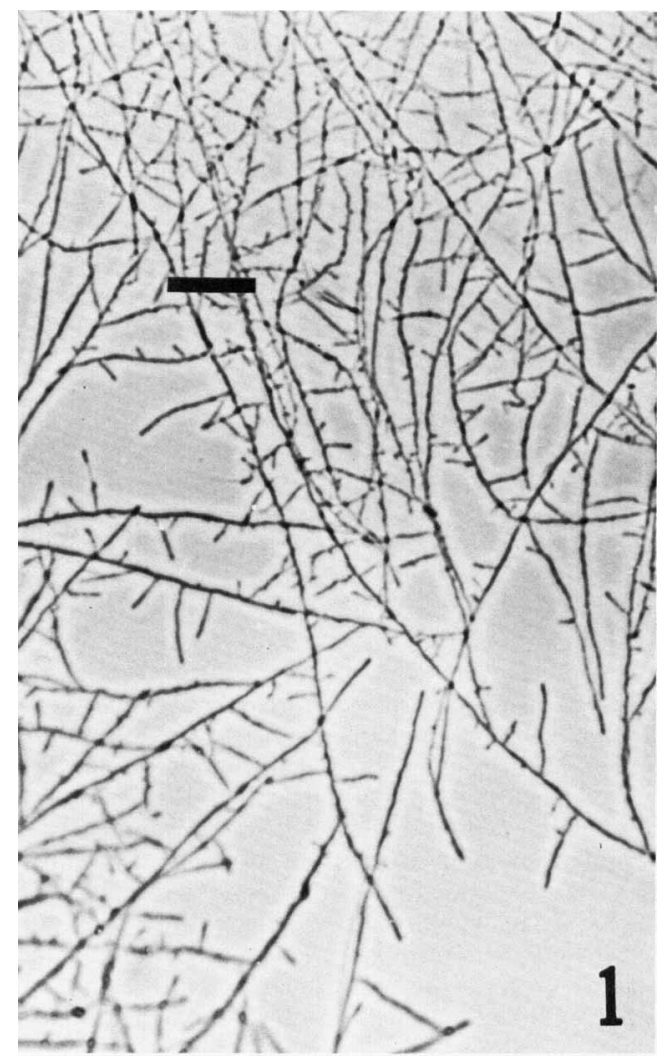

FIG. 1. Micrograph of an edge of a colony of strain $\mathrm{SK} \& \mathrm{~F}-\mathrm{AAA} 025^{\mathrm{T}}$ with unfragmented vegetative mycelium (10-day-old culture on TPC). Bar $=20 \mu \mathrm{m}$.

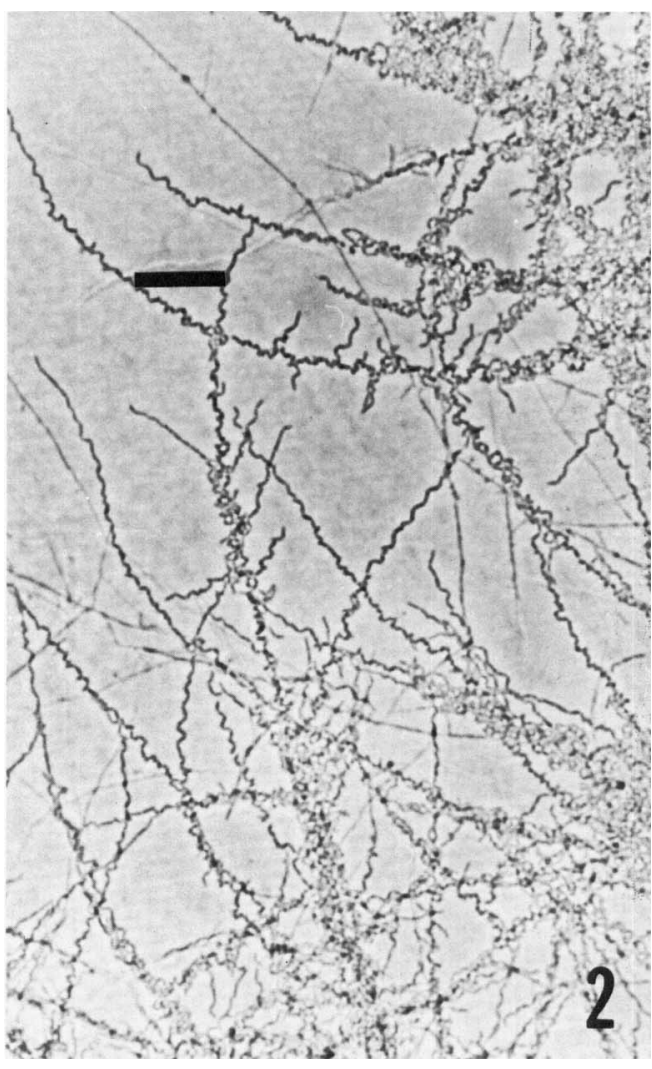

FIG. 2. Micrograph of an edge of a colony of strain SK\&F-AAA $025^{\mathrm{T}}$ with fragmented vegetative mycelium (10-day-old culture on TPC). Bar $=20 \mu \mathrm{m}$.

carefully removed from a plate and transferred to fresh plates, both colony types, along with intermediate colony types, were again observed.

Vegetative mycelium. The vegetative mycelium was well developed. Individual hyphae were long, moderately to densely branched, and about 0.5 to $1.0 \mu \mathrm{m}$ in diameter. On any one plate of TPC there were usually areas where the vegetative mycelium had fragmented into short rods and coccoid elements, as well as other areas where the vegetative mycelium appeared to be relatively unfragmented and stable (Fig. 1 through 3).

Extensive microscopic observations of the development of the two basic colony types on plates containing M172, TPC, and soil extract agar revealed that the vegetative mycelia of the yellow, butyrous colonies began to fragment after approximately 4 days on all three media. On plates containing TPC and soil extract agar the vegetative mycelia of the yellowish brown, leathery colonies appeared to be relatively unfragmented after 21 days; a few zig-zag hyphae and numerous gaps of the types described by Uesaka (23) were present. However, on plates 


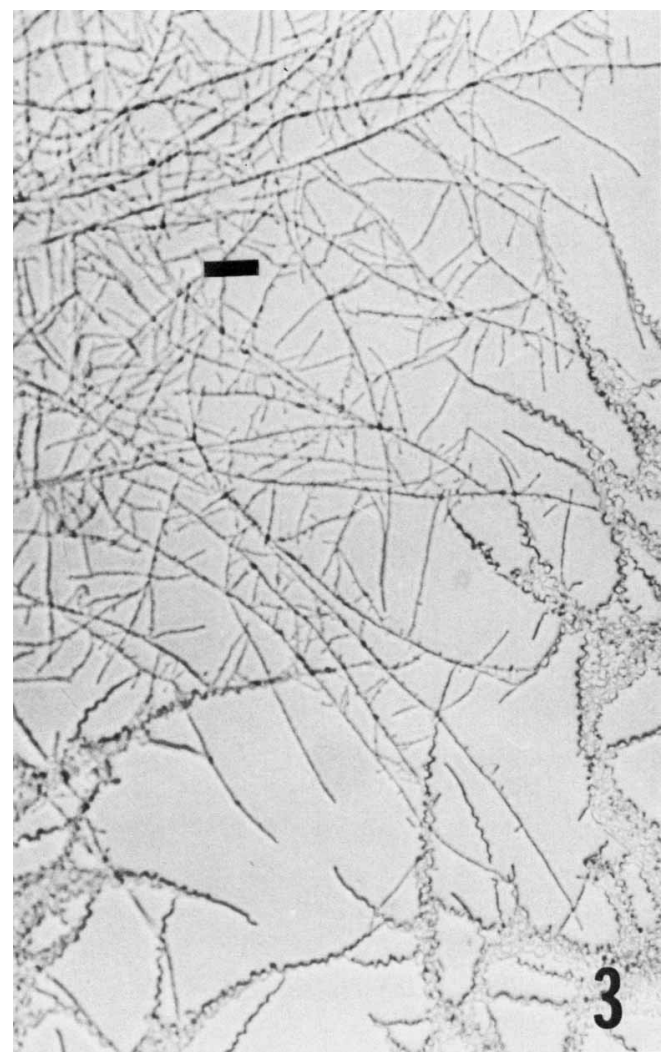

FIG. 3. Micrograph of vegetative mycelium of strain SK\&F-AAA025 $5^{\mathrm{T}}$ at intersection of colonies (4day-old culture on TPC). Bar $=20 \mu \mathrm{m}$.

containing M172, the vegetative mycelia of the yellowish brown, leathery colonies usually fragmented after 4 to 6 days.

Aerial mycelium. The aerial hyphae were either unbranched or moderately and irregularly branched; they were either straight or irregularly curved. These hyphae frequently showed nocardoid zig-zags during sporulation (8) (Fig. 4) and completely fragmented into spores (Fig. 5), which were somewhat irregular in length (approximately 0.7 by 1.0 to $2.0 \mu \mathrm{m}$ ). The spores had smooth surfaces (Fig. 6).

Growth in submerged cultures. Strain SK\&FAAA025 $5^{\mathrm{T}}$ fragmented when it was grown in a submerged culture. Growth harvested from shaken flasks of tryptone-yeast extract broth on day 4 consisted largely of very short to relatively long rods and coccobacillary units. Branched hyphae were rarely observed.

Physiological and biochemical characteristics. Strain SK\&F-AAA025 $5^{\mathrm{T}}$ did not grow under anaerobic conditions. The temperature range for growth was 15 to $45^{\circ} \mathrm{C}$. Starch, casein, L-tyrosine, and hypoxanthine were hydrolyzed, but adenine and xanthine were not. Nitrate was reduced to nitrite. Milk was peptonized. Gelatin was both hydrolyzed and liquefied. Growth occurred in lysozyme broth. No hydrogen sulfide or melanoid pigments were produced. Esculin and hippurate were decomposed, but urea and allantoin were not. Catalase and deoxyribonuclease were produced. No growth occurred on $4 \% \mathrm{NaCl}$. Acid was produced from L-arabinose, D-cellobiose, dextrin, dextrose, D-fructose, Dgalactose, glycerol, glycogen, i-inositol, lactose, maltose, D-mannose, D-melezitose, melibiose, $\alpha$-methyl-D-mannoside, D-ribose, salicin, starch, sucrose, trehalose, and D-xylose. No acid was produced from i-erythritol, inulin, or Lsorbose. Acid production during an initial culture in medium containing adonitol, dulcitol, Dmannitol, $\alpha$-methyl-D-glucoside, raffinose, rhamnose, or D-sorbitol was variable. However, when the strain was subcultured in media containing these carbohydrates, the results were consistent; acid was produced from $\alpha$-methyl-Dglucoside and raffinose but not from adonitol, dulcitol, D-mannitol, rhamnose, or D-sorbitol.

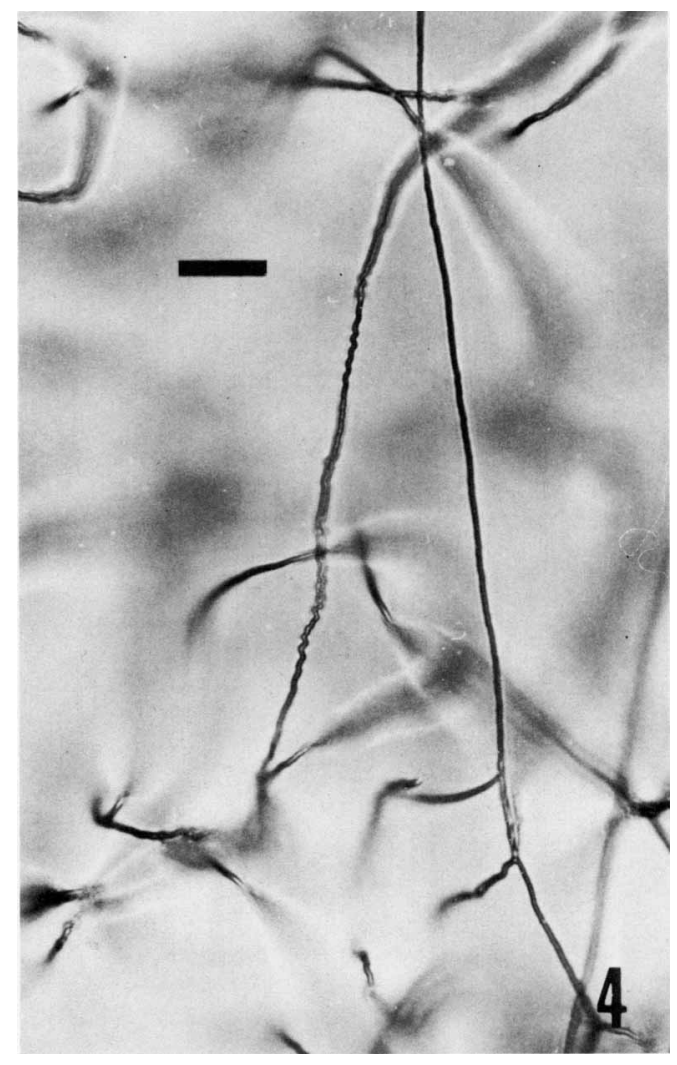

FIG. 4. Micrograph of aerial hyphae of strain SK\&F-AAA025 ${ }^{\mathrm{T}}$ showing the nocardoid zig-zag which occurs frequently during spore formation. Bar $=20$ $\mu \mathrm{m}$. 


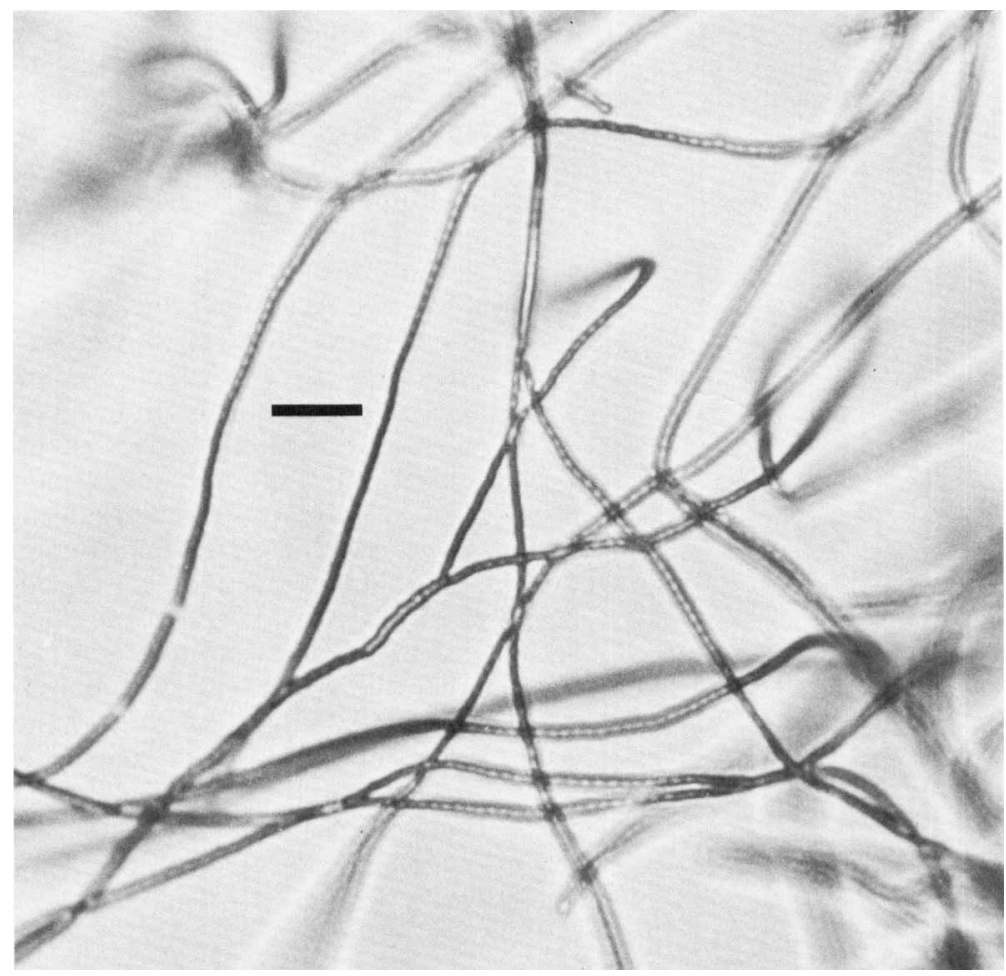

FIG. 5. Micrograph of sporulating aerial hyphae of strain SK\&F-AAA025 ${ }^{\mathrm{T}}$ (33-day-old culture on soil extract agar). Bar $=10 \mu \mathrm{m}$.

Citrate, malate, succinate, acetate, pyruvate, propionate, and lactate were utilized. Oxalate, tartrate, and benzoate were not utilized. During an initial culture in medium containing formate, utilization was variable; when the strain was

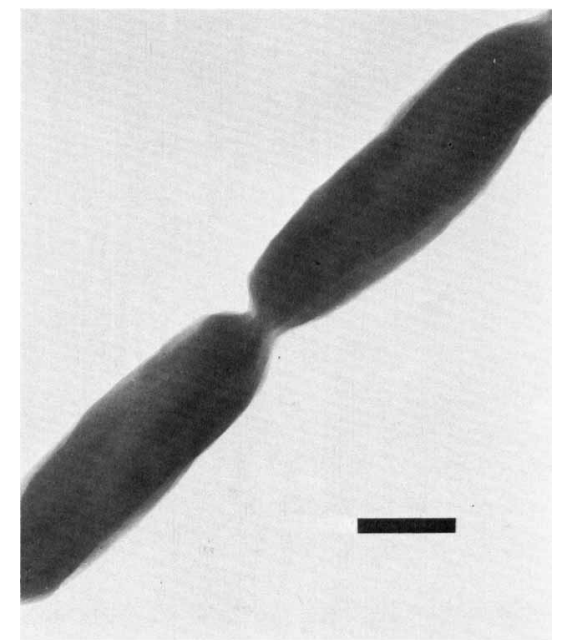

FIG. 6. Electron micrograph illustrating smooth spores of strain SK\&F-AAA025 ${ }^{\mathrm{T}}$ (19-day-old culture on soil extract agar). Bar $=1 \mu \mathrm{m}$. subcultured, the results were consistently negative.

Chemotaxonomy. Purified cell wall preparations of strain SK\&F-AAA025 $5^{\mathrm{T}}$ contained mesodiaminopimelic acid, alanine, glutamic acid, glucosamine, and muramic acid; no diagnostic sugars were present. Whole-cell hydrolysates contained galactose, glucose, mannose, ribose, and rhamnose; madurose was absent. The phospholipids found were cardiolipin, diphosphatidylglycerol, lysocardiolipin, phosphatidylethanolamine, phosphatidylethanolamine acylated with hydroxy fatty acids, phosphatidylinositol, and unknown glucosamine-containing phospholipids. Thus strain SK\&F-AAA025 $5^{\mathrm{T}}$ has a type III cell wall (13) with a type $C$ whole-cell sugar pattern (13) and a type PIV phospholipid composition (11). No mycolic acids of any type were found in the cell extracts.

Whole-cell hydrolysates were prepared from relatively stable cultures of each of the two basic colonial morphology variants. Both of these hydrolysates contained meso-diaminopimelic acid and had a type $C$ sugar pattern (13).

Appearance on various media. All plates were incubated for 3 weeks at $28^{\circ} \mathrm{C}$ in the dark. The colors of the cultures were determined by comparison with chips from the ISCC-NBS Centroid 
Color Charts $(7,18)$ or the Munsell Book of Color (17). The characteristics of the culture on the media tested are given below.

Yeast extract-malt extract agar: growth excellent, raised and wrinkled; vegetative mycelium yellow (ISCC-NBS 89, pale yellow); reverse yellow brown; aerial mycelium abundant, white (ISCC-NBS 263, white); no soluble pigment present.

Inorganic salts-starch agar: growth good; vegetative mycelium yellow (ISCC-NBS 82, vivid yellow); reverse yellow brown; aerial mycelium abundant but thin, white (ISCC-NBS 263, white); light brown soluble pigment variably present.

Oatmeal agar: growth fair to good; vegetative mycelium yellow (ISCC-NBS 89, pale yellow); reverse yellow brown; aerial mycellium abundant but thin, white (ISCC-NBS 263, white); no soluble pigment present.

Glycerol-asparagine agar: growth good; vegetative mycelium yellow (ISCC-NBS 67, brilliant orange yellow); reverse yellow brown; aerial mycelium abundant but thin, white (ISCC-NBS 263 , white); light brown soluble pigment variably present.

M172: growth excellent, raised and wrinkled; vegetative mycelium yellow (ISCC-NBS 72, dark orange yellow); reverse yellow brown; aerial mycelium moderate, white (ISCC-NBS 263, white); yellow brown soluble pigment present.

Soil extract agar: growth fair, flat; vegetative mycelium yellow (ISCC-NBS 89, pale yellow); reverse yellow brown; aerial mycelium abundant but thin, white (ISCC-NBS 263, white); no soluble pigment present.

Tyrosine agar: growth good; vegetative mycelium yellow (ISCC-NBS 86, light yellow); reverse yellow brown; aerial mycelium abundant, white (ISCC-NBS 263, white); no soluble pigment present.

Defined agar: growth good, raised and wrinkled; vegetative mycelium yellow (ISCC-NBS 71 , moderate orange yellow); reverse yellow brown; aerial mycelium none to sparse, white (ISCC-NBS 263, white); yellow brown soluble pigment present.

Glucose-yeast extract agar: growth good, raised and wrinkled; reverse yellow brown; aerial mycelium none to sparse, white; light yellow brown soluble pigment present.

Nutrient agar: growth fair, thin; reverse light yellow brown; aerial mycelium none to sparse, white; no soluble pigment present.

Czapek-sucrose agar: growth fair, flat; reverse light yellow brown; aerial mycelium sparse, white; light yellow brown soluble pigment present.

TPC: growth fair to good; vegetative mycelium yellow (ISCC-NBS 86, light yellow); reverse yellow brown; aerial mycelium abundant, white (ISCC-NBS 263, white); no soluble pigment present.

Strain SK\&F-AAA025 ${ }^{\mathrm{T}}$ was photochromogenic. The aerial mycelium was white on all media when the cultures were grown in the dark. However, on at least three of the media used in the description of this strain (TPC, tyrosine agar, and oatmeal agar) the aerial mycelium was pale orange yellow (Munsell 10YR 9/2) when cultures were grown in the light.

Susceptibility to penicillin and rifampin. Strain SK\&F-AAA025 $5^{\mathrm{T}}$ was resistant to penicillin (10 U). Rifampin $(5 \mu \mathrm{g})$ produced zones of inhibition varying from 10 to $20 \mathrm{~mm}$ in diameter. However, these zones always contained satellite colonies, indicating that resistance developed readily.

\section{DISCUSSION}

Strain SK\&F-AAA025 ${ }^{\mathrm{T}}$ belongs to the group of organisms described as nocardioform by Prauser (19). Nocardioform actinomycetes reproduce solely by fragmentation of either all of their hyphae, or more or less accidently involved parts of their hyphae, into irregular to rodlike to coccoid elements. However, the generic placement of strain SK\&F-AAA025 $5^{\mathrm{T}}$ presents problems. Before the introduction of chemotaxonomy, this organism would most likely have been placed in the genus Nocardia because of the extensive fragmentation which occurs in its vegetative mycelium, but its type III cell wall and complete lack of mycolic acids now preclude this grouping.

On the basis of chemotaxonomy alone, the creation of a new genus might have been justified, as no nocardioform organism with the combination of a type III cell wall and a type PIV phospholipid pattern has been described previously. This was considered unwise because only one strain was available for description and this one strain possesses no distinctive reproductive structures or other morphological features that would make the new genus either easy to describe definitively or easy to recognize readily. However, strain SK\&F-AAA025 $5^{\mathrm{T}}$ can be accommodated in the genus Nocardiopsis as described by Meyer (15), and it is this more conservative approach that we have taken. Strain SK\&F-AAA025 ${ }^{\mathrm{T}}$ was compared with the one species previously placed in this genus (22), Nocardiopsis dassonvillei, by using the neotype strain, N. dassonvillei ATCC 23218 (15). Although the most obvious difference between these two organisms was the variety of colony morphologies that were usually present in plate cultures of strain SK\&F-AAA025 ${ }^{\mathrm{T}}$, they also differed in several other morphological, physiological, and chemotaxonomic characteristics (Table 1). Therefore, we regard strain SK\&F- 
TABLE 1. Comparison of some morphological, physiological, and chemotaxonomic characteristics of SK\&F-AAA025 ${ }^{\mathrm{T}}$ and $N$. dassonvillei

\begin{tabular}{lcc}
\hline \multicolumn{1}{c}{ Characteristic } & $\begin{array}{c}\text { Strain SK\&F- } \\
\text { AAA025 }\end{array}$ & N. dassonvillei \\
\hline $\begin{array}{l}\text { Growth at } 45^{\circ} \mathrm{C} \\
\text { Decomposition of: }\end{array}$ & + & $-(16)^{a}$ \\
$\quad$ Adenine & - & $+(16)$ \\
$\quad$ Xanthine & - & $+(16)$ \\
$\begin{array}{l}\text { Acid from: } \\
\text { i-Inositol }\end{array}$ & + & $-(16)$ \\
Lactose & + & $-(16)$ \\
Melezitose & + & $-(16)$ \\
Resistance to & Resistant & $2 \%$ Of 46 \\
lysozyme & & strains re- \\
& & sistant \\
& & $(16)$ \\
Growth in sub- & Almost complete & Rarely frag- \\
merged culture & fragmentation & ments (20) \\
Phospholipid type & PIV & PIII (11) \\
\hline
\end{tabular}

${ }^{a}$ The numbers in parentheses are reference numbers.

AAA $025^{\mathrm{T}}$ as a new species, for which we propose the name Nocardiopsis mutabilis (mu.ta' bi.lis. L. adj. mutabilis changeable, variable, inconstant, referring to the variety of colony morphology observed, particularly on rich organic media). Strain SK\&F-AAA025, the type strain of N. mutabilis, has been deposited in the American Type Culture Collection, Rockville, Md., as strain ATCC 31520.

\section{ACKNOWLEDGMENTS}

We are deeply indebted to Mary P. Lechevalier for the electron microscopy, as well as the phospholipid and pure cell wall analyses. Of even greater value was her advice and encouragement while we were working with this very interesting but difficult organism.

\section{LITERATURE CITED}

1. American Type Culture Collection. 1980. Catalogue of strains I, 14th ed. American Type Culture Collection, Rockville, Md.

2. Becker, B., M. P. Lechevalier, and H. A. Lechevalier. 1965. Chemical composition of cell-wall preparations from strains of various form-genera of aerobic actinomycetes. Appl. Microbiol. 13:236-243.

3. Gordon, R. E. 1966. Some criteria for the recognition of Nocardia madurae (Vincent) Blanchard. J. Gen. Microbiol. 45:355-364.

4. Gordon, R. E. 1967. The taxonomy of soil bacteria, p. 293-321. In T. R. G. Gray and D. Parkinson (ed.), The ecology of soil bacteria. Liverpool University Press, Liverpool.
5. Gordon, R. E., and J. M. Mihm. 1962. Identification of Nocardia caviae (Erikson) nov. comb. Ann. N.Y. Acad. Sci. 98:628-636.

6. Higgins, M. L., M. P. Lechevalier, and H. A. Lechevalier. 1967. Flagellated actinomycetes. J. Bacteriol. 93:14461451.

7. Kelly, K. L., and D. B. Judd. 1976. Color: universal language and dictionary of names. Special publication 440. National Bureau of Standards, Washington, D.C.

8. Lechevalier, H. A., and M. P. Lechevalier. 1970. A critical evaluation of the genera of aerobic actinomycetes, p. 393 405. In H. Prauser (ed.), The Actinomycetales. Gustav Fischer Verlag, Jena.

9. Lechevalier, H. A., M. Solotorovsky, and C. I. McDurmont. 1961. A new genus of the Actinomycetales: Micropolyspora gen. nov. J. Gen. Microbiol. 26:11-18.

10. Lechevalier, M. P. 1968. Identification of aerobic actinomycetes of clinical importance. J. Lab. Clin. Med. 71:934-944.

11. Lechevalier, M. P., C. DeBièvre, and H. Lechevalier. 1977. Chemotaxonomy of aerobic actinomycetes: phospholipid composition. Biochem. Syst. Ecol. 5:249-260.

12. Lechevalier, M. P., and H. Lechevalier. 1957. A new genus of the Actinomycetales: Waksmania gen. nov. J. Gen. Microbiol. 17:104-111.

13. Lechevalier, M. P., and H. Lechevalier. 1970. Chemical composition as a criterion in the classification of aerobic actinomycetes. Int. J. Syst. Bacteriol. 20:435-443.

14. Lechevalier, M. P., H. Lechevalier, and A. C. Horan. 1973. Chemical characteristics and classification of nocardiae. Can. J. Microbiol. 19:965-972.

15. Meyer, J. 1976. Nocardiopsis, a new genus of the order Actinomycetales. Int. J. Syst. Bacteriol. 26:487-493.

16. Mishra, S. K., R. E. Gordon, and D. A. Barnett. 1980 Identification of nocardiae and streptomycetes of medical importance. J. Clin. Microbiol. 11:728-736.

17. Munsell Color Co., Inc. 1973. Munsell book of color, neighboring hues edition, matte finish collection. Munsell Color Co., Inc., Baltimore.

18. National Bureau of Standards. 1955. ISCC-NBS centroid color charts. Supplement to NBS circular 553. Standard sample 2106. Office of Standard Reference Materials, National Bureau of Standards, Washington, D.C.

19. Prauser, H. 1974. Host-phage relationships in nocardioform organisms, p. 84-85. In G. H. Brownell (ed.), Proceedings of the First International Conference on the Biology of the Nocardiae, August 12-15, 1974, Merida, Venezuela. McGowen Printing Co., Augusta, Ga.

20. Prauser, H. 1976. New nocardioform organisms and their relationships, p. 193-207. In T. Arai (ed.), Actinomycetes: the boundary microorganisms. University Park Press, Baltimore.

21. Shirling, E. B., and D. Gottlieb. 1966. Methods for characterization of Streptomyces species. Int. J. Syst. Bacteriol. 16:313-340.

22. Skerman, V. B. D., V. McGowan, and P. H. A. Sneath (ed.). 1980. Approved lists of bacterial names. Int. J. Syst. Bacteriol. 30:225-420.

23. Uesaka, I. 1969. Breaking up of vegetative mycelium of Nocardia and Streptomyces cultures on agar film. Jpn. J. Microbiol. 13:65-77.

24. Waksman, S. A. 1961. The actinomycetes, vol. 2. The Williams \& Wilkins Co., Baltimore. 